\title{
Associative Learning Modifies Two Behaviors in the Leech, Hirudo medicinalis
}

\author{
Christie L. Sahley ${ }^{1}$ and Donald F. Ready ${ }^{2}$ \\ ${ }^{1}$ Department of Biology, Yale University, New Haven, Connecticut 06511, and 'Department of Biology, Purdue University, \\ West Lafayette, Indiana 47907
}

\begin{abstract}
We report that 2 behaviors, stepping and shortening, are modified by associative learning in the leech, Hirudo medicinalis. Experiment 1 explored conditioning of the "stepping" response. Paired presentations of touch to the medial dorsal surface of the leech and shock to the tail of the leech resulted in the development of stepping to the touch. Leeches in control groups experiencing the CS alone, US alone, or explicitly unpaired presentations of the CS and US did not. In experiments 2-4, classical conditioning explored conditioning of the touch-elicited shortening reflex. We found that the reflex was enhanced following paired CS-US presentations but not following CS alone, US alone, or explicitly unpaired presentations of the stimuli. Moreover, the learning was extinguished following 15 unreinforced presentations of the CS but was retained for at least $24 \mathrm{hr}$ without extinction training. Moreover, the associative effect was not evident when the CS and US were presented in a backward relationship. That is, no learning was observed when the US preceded the CS. Lastly, the hand-held stimuli were replaced with implanted electrodes. Using a $3 \mathrm{~V}$ pulse that mimicked the touch stimulus (CS), we found that paired CS-US presentations produced a significant enhancement in the shortening reflex. Again, no enhancement was observed following unpaired $C S$, US presentations.
\end{abstract}

The study of the cellular basis of associative learning in invertebrates has been dominated by the study of molluscs and, but for a few exceptions, Drosophila melanogaster (Dudai et al, 1976; Dudai, 1977, 1985; Quinn et al., 1979; Tempel et al., 1984; Tully, 1984, 1987) and Apis melifera (Couvillon and Bitterman, 1980, 1982; Erber et al., 1980; Erber, 1981, 1984; Bitterman et al., 1983; Menzel, 1984) being the 2 most noteworthy, the current understanding of the cellular and molecular events underlying learning come from studies on molluscs, including Aplysia californica and Hermissenda crassicornus (for reviews, see Farley and Alkỏn, 1985; Mpitsos and Lukowiak, 1985; Carew and Sahley, 1986; Byrne, 1987). It is clear from studies of these animals that common themes both on the behavioral and mechanistic levels have emerged. For example, associative

\footnotetext{
Received Oct. 23, 1987; revised May 18, 1988; accepted May 20, 1988.

We wish to thank Dr. Jerry Rudy for helpful comments and criticisms of the manuscript. In addition, we would like to thank Dr. Jerry Rudy and Dr. Don Weisz for numerous discussions of the data. This work was supported by R01MH37902, Whitehall Foundation Grant 56840, and a Sloan Fellowship to C.L.S. and NIH PHS NS 16515 and Sloan BR2040 to D.F.R.

Correspondence should be addressed to Dr. Christie L. Sahley, Department of Biology, Yale University, Box 6666, New Haven, CT 06511.

Copyright (C) 1988 Society for Neuroscience $0270-6474 / 88 / 124612-09 \$ 02.00 / 0$
}

learning in both species is dependent on the contingent as well as the contiguous relationships between stimuli (Sahley et al., 1981a, b; Colwill, 1985; Hawkins et al., 1986; Farley, 1987a), just as is learning in vertebrates (Rescorla, 1968, 1969; Kamin, 1969). Moreover, on the mechanistic level, the identified learning-dependent changes involve second-messenger modulation of $\mathrm{K}^{+}$channels of sensory neurons (Klein et al., 1982; see Crow, 1988 , for a review). No data, however, address the generality of these common themes in other invertebrate phyla. Will contiguity and contingency be as important in the formation of associations in other invertebrates? Will learning-dependent changes in behavior be the reflection of similar or dissimilar cellular mechanisms? For these reasons, we have decided to extend the study of the neural basis of learning to a nonmolluscan invertebrate, the annelid Hirudo medicinalis.

The leech has proved to be a useful preparation in neurobiology and has been advantageous for the study of synaptic physiology (Nicholls and Purves, 1970; Muller and Nicholls, 1973; Muller and Scott, 1981), neurodevelopment (Shankland and Weisblat, 1984; Kramer and Weisblat, 1985; Stent and Weisblat, 1985; Stewart et al., 1985, 1986; Loer et al., 1986), regeneration (Baylor and Nicholls, 1969; Jansen and Nicholls, 1972; Ready and Nicholls, 1979; Scott and Muller, 1980; Muller and Nicholls, 1981; DeRiemer et al., 1983; Macagno et al., 1985; French and Muller, 1986), as well as the cellular mechanisms underlying several behaviors, including swimming (Kristan et al., 1974; Ort et al., 1974; Kristan and Calabrese, 1976; Friesen and Stent, 1978; Friesen et al., 1978; Weeks and Kristan, 1978; Weeks, 1982a, b; Kristan, 1983; Friesen, 1985; Nusbaum, 1986; Nusbaum and Kristan, 1986), stepping (inch-worm crawling) (Stern-Tomlinson, et al., 1986a, b), feeding (Galur and Kindler, 1966; Dickinson and Lent, 1984), bending (Kristan, 1982; Kristan et al., 1982), and shortening (Magni and Pellegrino, 1978a, b; Kramer, 1981). More recently, the leech has been used to study neuronal (Belardetti et al., 1982; Biondi et al., 1982) and behavioral plasticity, including habituation, sensitization (Debski and Friesen, 1985; Lockery and Kristan, 1985; Lockery et al., 1985; Stoller and Sahley, 1985; Boulis and Sahley, 1988) and classical conditioning (Henderson and Strong, 1972; Sahley and Ready, 1985). We report that 2 behaviors, stepping and shortening, are modified by associative learning.

Stepping, or inch-worm crawling, recently has been characterized by Stern-Tomlinson and colleagues (1986a, b). Briefly, stepping consists of lifting the anterior sucker, rostral-caudal extension of the body, replanting of the anterior sucker, lifting of the posterior sucker, rostral-caudal contraction of the body, accompanied by the reattachment of the posterior sucker in close apposition to the anterior sucker. This results in the character- 
istic "looping" behavior that distinguishes inch-worm crawling from veriform crawling.

Shortening is a defensive withdrawal response to either a tactile or a photic stimulus applied to the head of the leech. That is, either stimulus elicits an immediate and simultaneous contraction of the longitudinal muscles in the leech, resulting in a dramatic "shortening" of the length of the leech. It has recently been established that this reflex can be modified by experience. That is, repeated presentations of a light tactile stimulus or a photic stimulus result in a decrement or habituation of the response (Lockery et al., 1985; Stoller and Sahley, 1985). A shock presented to the animal following habituation training produces a marked restoration or dishabituation of the response (Stoller and Sahley, 1985). Likewise, a shock applied to the leech prior to habituation signiticantly retards the development of habituation (Stoller and Sahley, 1985; Boulis and Sahley, 1988). In this paper, we extend our analysis of plasticity by demonstrating that this shortening reflex can be modified by associative learning.

\section{Experiment 1}

\section{Classical conditioning of the stepping response}

The goal of Experiment 1 was to test whether stepping could be modifed by associative learning. Specifically, the question was: Can the pairing operations that produce classical conditioning in other animals, including invertebrate as well as vertebrate species (see Carew and Sahley, 1986; Sahley, 1984, for reviews), result in classical associative learning in the leech. We selected a light tactile stimulus applied to the dorsal surface of the leech as the conditioned stimulus (CS) because this stimulus never results in stepping. The unconditioned stimulus (US), a shock applied to the tail, reliably produces 3-5 stepping cycles. The question was whether leeches learn to associate a light tactile stimulus to the back with a shock to the tail such that a test tactile stimulus to the back of the leech will result in at least one stepping cycle.

\section{Materials and methods \\ Animals}

Hirudo medicinalis were obtained from Ricarimpex, Bordeaux, France. Approximately 1 week after arrival in the lab, 40 leeches were randomly selected and placed in individual Plexiglas containers $[21.6$ (1) $\times 5.5$ (w) $\times 6(\mathrm{~d}) \mathrm{cm}$ ] in about $2 \mathrm{~cm}$ of artificial spring water at room temperature. Training began after the leeches had been isolated for $5 \mathrm{~d}$, during which time they were left undisturbed. Training and testing lasted $1 \mathrm{~d}$. During this period, the leeches were maintained on a 12/12 lightdark cycle. The leeches remained in their training chambers throughout the duration of the experiment.

\section{Stimulus materials}

The CS was provided by a heat-pulled segment of tygon tubing attached to a wooden handle. The delivery of the stimulus was similar to that described by Debski and Friesen (1985) and consisted of a light 1 sec stroke of the body with the CS in an anterior-to-posterior direction. Each stroke crossed 3 annuli. The US was a 20 V 500 millisecond shock delivered via a bipolar electrode spanning (but not in direct contact with) the tail of the leech. The shock stimulus was provided by a Grass stimulator (model S88) and delivered to the leech via a Grass stimulation isolation unit (model SIU).

\section{Experimental groups}

Forty leeches were randomly assigned to 1 of 4 experimental groups as follows.

Paired group $(P)$. Leeches in the paired group $(n=10)$ received 20 paired presentations of the CS and US with the CS preceding the US.

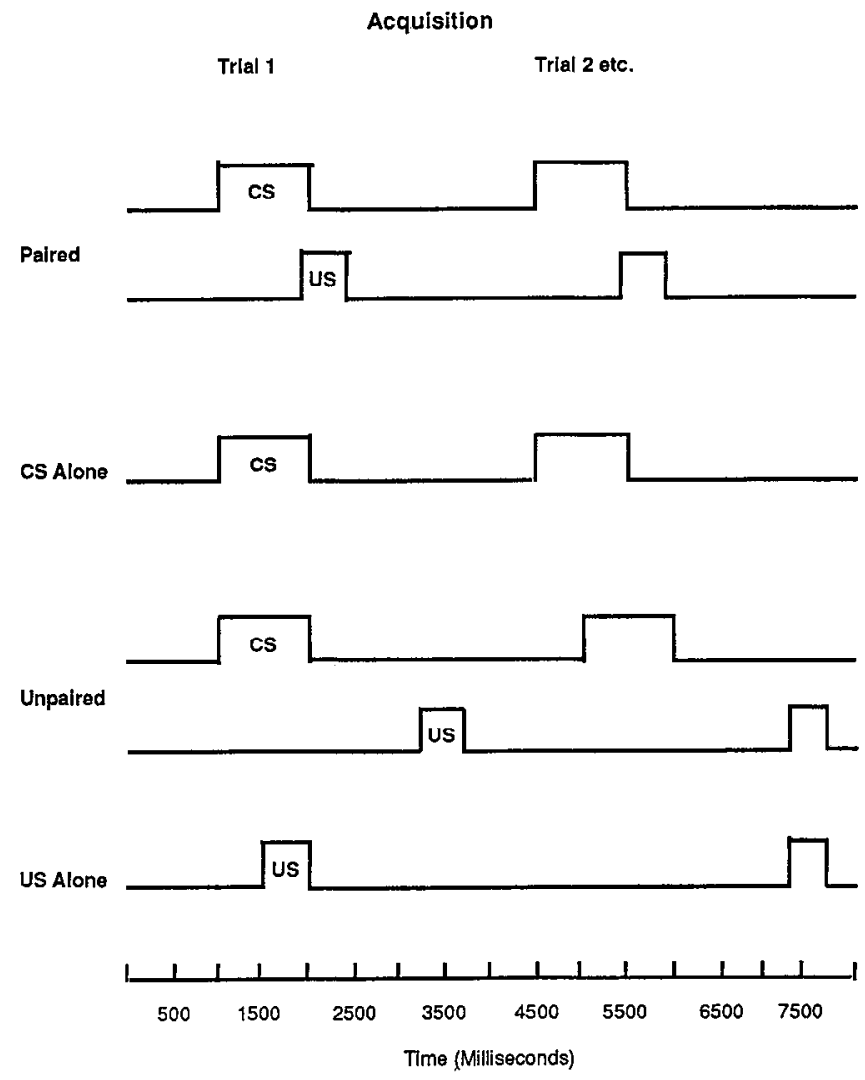

Figure 1. Schematic representation of the onset, offset, and temporal relationship of the stimuli used for the training regimen for Experiment 1. Leeches were randomly divided into 4 groups: CS alone, US alone, Unpaired, and Paired presentations of the CS and US.

The pairings were separated by approximately $2.5 \mathrm{~min}$ intertrial intervals. Testing, consisting of 3 CS-alone presentations, was done $20 \mathrm{~min}$ following training.

Explicitly unpaired group (UP). Leeches in this group $(n=10)$ received the same stimuli but separated by a $1.25 \mathrm{~min}$ interstimulus interval. As for leeches in the paired group, testing occurred 20 min following delivery of the 20th USS.

$C S$ alone or US alone. Leeches in these groups ( $n=10$ each group) were treated as described for the animals in the paired group except that they were presented with only the tactile stimulus (CS) or the shocks (US), respectively, at $2.5 \mathrm{~min}$ intervals. Testing occurred $20 \mathrm{~min}$ after the delivery of the last stimulus.

A schematic representation of the training protocol for the four groups is presented in Figure 1.

\section{Dependent measure}

Animals were video taped using a Panasonic camera and VCR. The animals were arranged prior to testing so that the experimenter did not know the experimental history of each animal. Likewise, scoring was done without the knowledge of the experimental history of each leech.

Each leech received 3 test trials separated by $2.5 \mathrm{~min}$ interstimulus intervals following training. If an animal stepped to the test stimulus the performance was scored as positive $(+)$. If no step occurred the animal was scored as negative $(-)$. If an animal stepped on 2 of the 3 trials, it was considered to have demonstrated a conditioned response (CR). A step was defined as the lifting of the anterior sucker, extension of the body, reattachment of the anterior sucker, and contraction of the body.

\section{Statistical analysis}

Because of the dichotomous nature of the data, a nonparametric test for significance of difference between two proportions was used to analyze the data (Bruning and Kintz, 1968). 


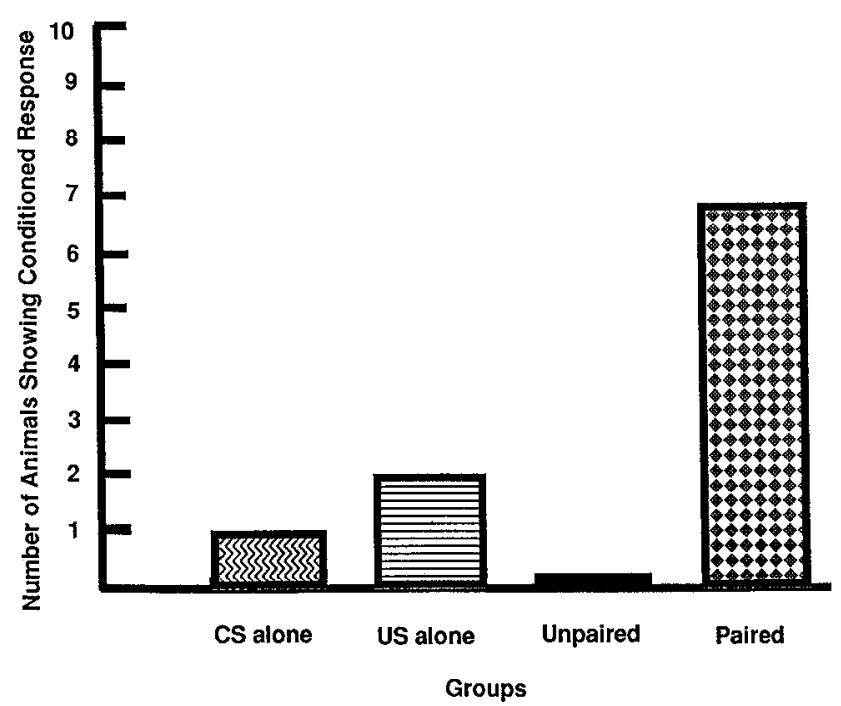

Figure 2. The number of leeches showing a conditioned response to the test stimulus following 20 training trials. Only leeches that received paired presentations of the CS and US showed an increased incidence of stepping to the touch stimulus.

\section{Results and discussion}

The number of animals in each group showing CRs is presented in Figure 2. It is apparent that only the leeches that experienced paired presentations of the touch and shock showed stepping to the test stimulus, 7 out of 10 leeches in this group showed a CR. In contrast, only 2 of the 10 leeches in the US alone group, 1 of the 10 leeches in the CS alone group, and 0 of the 10 in the Unpaired group showed a CR. The performance of leeches in the paired group was significantly different from the performance of the leeches in each of the control groups. A $z$ test for proportions indicated that the proportion of correct responses in the paired group was significantly different from the proportion of correct responses in each of the other groups $(z=3.68$, $p<0.001$, P vs US; $z=4.89, p<0.001$, $\mathrm{P}$ vs CS; $z=6.79, p$ $<0.001, \mathrm{P}$ vs UP). Leeches in the control groups experienced the same CS and US stimuli as those animals in the experimental group with one important exception; they either experienced only one of the stimuli (CS alone or US alone groups) or, when they experienced both (Unpaired group), never in a paired relationship. The observed CR to the test stimulus is a result of the pairing-produced learned association between the touch CS and the shock US and thus is an example of Pavlovian associative learning.

\section{Experiment 2}

\section{Classical conditioning of the shortening reflex}

The goal of this experiment is to determine if classical conditioning can modify the leech's defensive shortening response. We chose this behavior for several reasons. First, the shortening response is easily measured and quantified. Second, it is a reflex, and as such the underlying circuitry should prove simpler than that of the more complex stepping response. Moreover, many neurons within the circuit have been identified and characterized (Nicholls and Baylor, 1968; Laverick, 1969; Nicholls and Purves, 1970; Stuart, 1970; Bagnoli et al., 1975; Magni and Pellegrino, 1978a, b; Kristan et al., 1982), and this will facilitate the analysis of the underlying cellular mechanisms. Finally, in many respects the shortening response of the leech is very similar to the extensively studied gill-withdrawal reflex of the marine mollusc, Aplysia californica. Both are defensive-touch-elicited behaviors that can be modified by habituation and sensitization. Studies of plasticity of this reflex will allow direct comparisons into the generality of the cellular and molecular events underlying learning described in Aplysia. For example, will the same mechanisms that produce habituation and sensitization in molluscs share similarities with those observed in annelids or will completely different mechanisms be found?

Finally, on a more practical level, the nervous system of the leech is extremely amenable to cellular analysis and, as discussed in the introduction, has been used successfully to study a number of neurobiological phenomena. Moreover, most recently techniques have been developed to study single indentified cells or pairs of cells in culture (Ready and Nicholls, 1979; Fuchs et al., 1981, 1982; Dietzel et al., 1986; B. W. Adams, J. Nicholls, and R. Stewart, personal communication). Thus, the analysis of learning can proceed from the behavior in the intact animal, to semi-intact preparations, to isolated nervous system, and finally to single identified cells in culture quite readily.

\section{Materials and methods Animals}

Twenty-four leeches were randomly assigned to the 4 training groups. Each of the control groups had 6 leeches. The experimental group had 10 leeches so that following acquisition training, the leeches could be divided into 2 groups of 5 for the postacquisition retention and extinction tests (described more fully below). However, one leech in the paired group died during training. In addition, of the 9 remaining leeches in the paired group, one showed no evidence of learning with training and therefore was not included in either the retention or extinction groups.

\section{Stimulus materials}

The stimulus materials used in this experiment were identical to those used in Experiment 1.

\section{Procedures}

The procedures used in this experiment were similar to those used in Experiment 1 except that the CS was applied to the head of the leech.

\section{Training regimen}

Acquisition. All leeches received 30 training trials. The trials were separated by a 2 min intertrial interval. A single test trial, which consisted of a CS alone presentation, was interpolated into the training regimen every fifth trial. That is, after trials $5,10,15,20,25,30$, and 35 a test CS was presented in order to document the rate of acquisition.

Extinction. Twenty-four hours following the final test trial extinction training began. Leeches in the paired group were divided into 2 groups matched for performance. Leeches in one group (E) received extinction training consisting of $15 \mathrm{CS}$ alone trials at an intertrial interval (ITI) of 2 min. Leeches in the second group $(R)$ received no extinction training but rather were used to provide a control for possible forgetting over the $24 \mathrm{hr}$ interval. Testing occurred on trial 15. A schematic representation of the training regimen is presented in Figure 3.

\section{Dependent measure}

The dependent measure was the ratio of the length of the animal following the test stimulus to the length of the animal immediately preceding the presentation of the test stimulus. This ratio was then expressed as the percentage of initial contraction elicited by the test stimulus pretest.

\section{Statistical analysis}

An analysis of variance (ANOVA) was used to compare performance between the groups. Newman-Keuls post hoc tests were used for further analysis of the data when the $F$ ratios were significant (Keppel, 1973). A Student's $t$ test was used to compare the scores for animals in the Extinction and Retention groups. 


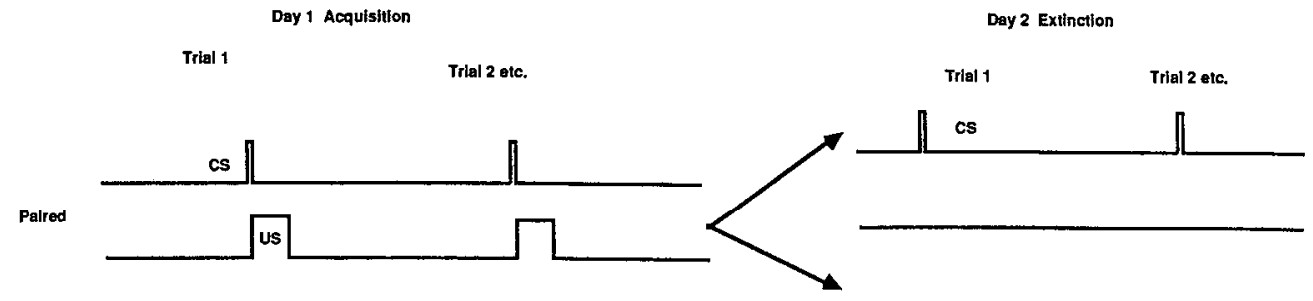

CS Alone

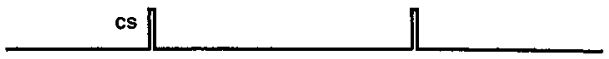

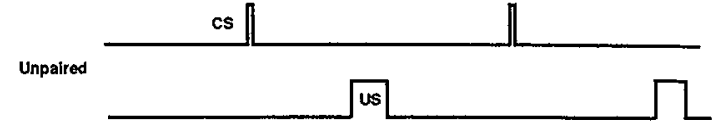

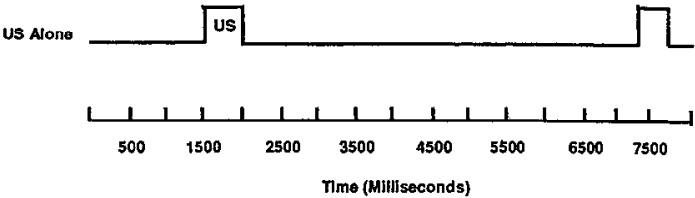

\section{Results and discussion}

The mean percentage of inital contraction across trials for animals in all groups is presented in Figure 4. The ANOVA on the acquisition phase revealed a significant group main effect, $F(3,23)=3.92, p<0.05$, and a significant trial effect, $F(6,138)$ $=3.22, p<0.01$. Since a significant group $\times$ trial interaction $F(18,138)=3.72, p<0.01$, was revealed we used post hoc tests to analyze how the performance of each of the groups differed across trials (Keppel, 1973). The Newman-Keuls post hoc analysis revealed an interesting pattern of results. As we have seen previously, leeches in the CS alone group show a rapid reduction in response amplitude across trials; their responding decreased to $20 \%$ by block 2 . Leeches in the Unpaired group did not show a significant decrease in responding until the block 3 test, and at this point responding in the Unpaired group was not different from responding in the CS alone group. Leeches in the US alone group did not show a significant decrease in responding until the block 4 test, but the responding for leeches in this group remained significantly greater than responding for leeches in the CS alone and Unpaired groups throughout training, $(p<0.05)$. Leeches in the Paired group maintained their responding until the block 5 test and then showed a significant increase in responding over the last 2 tests. Since an increase in responding across trials was observed only in the Paired group, we interpret this increase as the result of associative learning. The decrease in responding seen in the CS alone group reflects habituation to the repeated CS presentation. Likewise, the decrement in responding seen in the US alone and Unpaired groups is most likely due to habituation to the repeated presentations of the CS with the difference between the 2 groups most likely due to the differing number of CS presentations, 5 in the US alone group and 35 in the Unpaired group. The initial retardation of habituation is most likely due to the sensitizing effects of the US.

As is also seen in Figure 4, extinction of the learning was rapid and complete. Leeches experiencing the CS alone extinction
Figure 3. Schematic representation of the onset, offset, and temporal relationship of the stimuli used for the training regimen for Experiment 2. Leeches were randomly divided into 4 groups: CS alone, US alone, Unpaired, and Paired presentations of the CS and US. Following acquisition training and testing, leeches in the Paired group were randomly divided into 2 subgroups and either experienced extinction training or were held for retention. trials showed a dramatic reduction in their response magnitude as compared to the leeches in the retention group, which were simply held for the $24 \mathrm{hr}$ requircd for extinction training. Following extinction training leeches in the group $\mathrm{E}$ responded at $20 \%$ of their initial contraction magnitude. Leeches in the retention group showed no decrement in responding; their responding remained at $109 \%$ of baseline. A $t$ test indicated that this difference was significant, $t(6)=3.78, p<0.01$. This indicates that the decrease in responding was due to extinction and not forgetting.

In summary, we have shown that the shortening reflex can be modified by associative learning; paired presentations of touch and shock produced an enhanced shortening to test trial presentations of the CS alone. This learning is retained for at least $24 \mathrm{hr}$ and is extinguished by 10 repeated presentations of the CS.

\section{Experiment 3}

\section{Backward conditioning}

In most conditioning experiments, learning occurs easily when the presentation of the CS precedes the occurence of the US by at least a few seconds, and this is termed forward conditioning. In some instances, however, learning occurs when the CS follows the occurrence of the US. This is termed backward conditioning. Although backward associations are capable of being formed, it is reported that forward conditioning results in easier and faster formation of associations. In vertebrates conditioning of many skeletal responses is optimal when the CS precedes the US by about $500 \mathrm{msec}$. In contrast, little or no conditioning occurs when the stimuli are arranged in a backward relationship (see Kimble, 1961; Gormezano, 1972; Mackintosh, 1974, for reviews). Recent evidence indicates that the same principles of learning hold for at least one invertebrate species, Aplysia californica (Hawkins et al., 1986). Hawkins et al. (1986) tested the effects of several interstimulus intervals, including backward intervals, on the conditioning of the siphon-withdrawal response. They found that conditioning was optimal at the 0.5 
Figure 4. Mean percentage contraction, normalized to the initial contraction, of leeches in the 4 training groups across test trials is presented in Figure 4. Bars indicate the SEM. Comparison of the performance of leeches in all 4 training groups indicates that only the leeches in the Paired group showed an increase in responding to the touch stimulus. Leeches in all other groups showed a decrease in responding to the test stimulus.

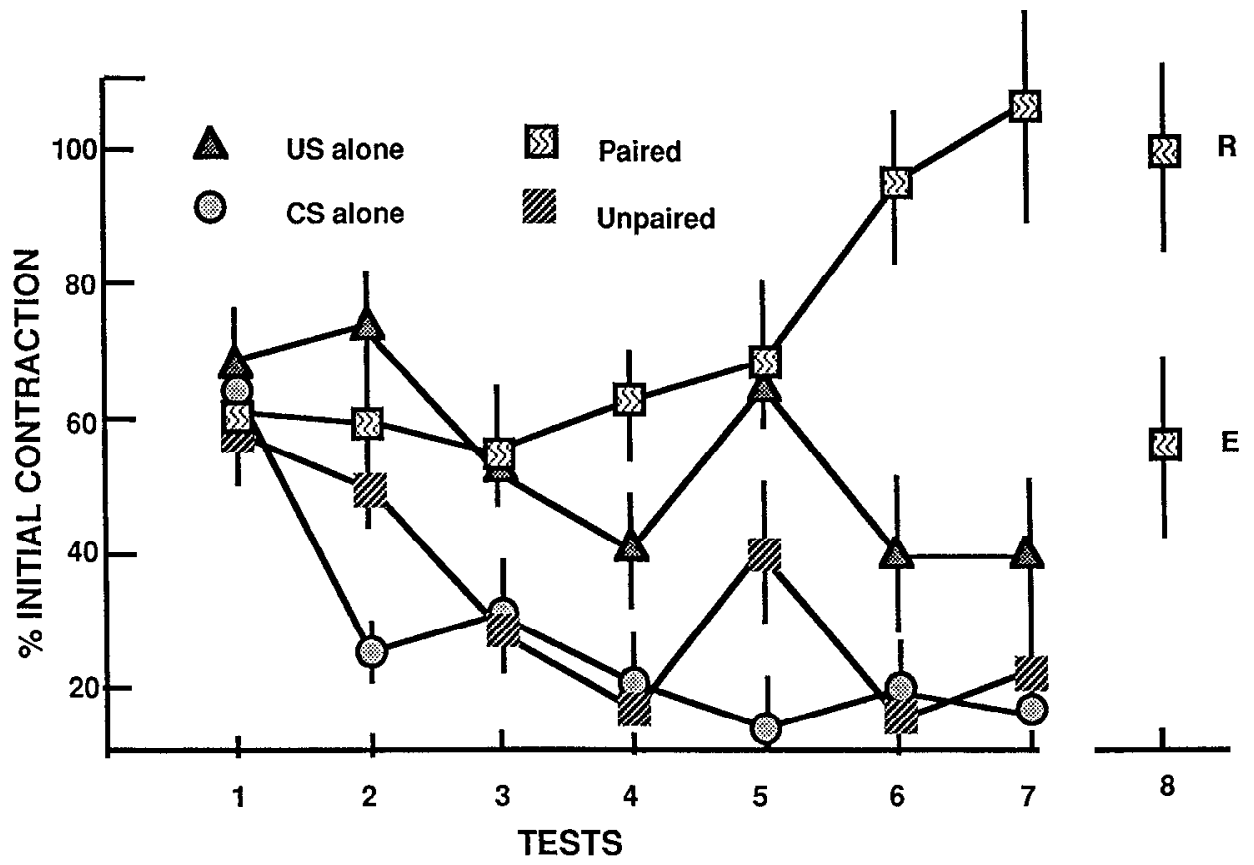

sec interval and that there was little or no conditioning at the other intervals. The goal of this experiment is to evaluate the leech's ability to form backward associations.

In addition, a secondary goal of this experiment was to replicate the learning observed in Experiment 2.

\section{Materials and methods}

The methods were exactly as described for Experiment 2 except that only 2 training regimens were used, Forward Paired and Backward Paired. Leeches in the Forward Paired group were treated exactly the same as leeches in the Paired group of Experiment 2. Leeches in the Backward Paired group received paired presentations of the CS and US except that the onset of the CS was coincident with the offset of the US. The dependent measure and statistical analysis were exactly as in Experiment 2.

\section{Results and discussion}

The mean percentage of initial contraction for animals in the Forward Paired and Backward Paired groups is presented in Figure 5. As seen, shown in Figure 5, and as revealed by the ANOVA and Newman-Keuls post hoc test, leeches in the Forward Paired group showed an increase in responding over trials, whereas leeches in the Backward Paired group showed a decrease in responding over trials: group effect, $F(1,8)=23.06, p<0.01$; group by block interaction, $F(6,48)=9.9, p<0.01$; NewmanKeuls, $p<0.01$. Leeches in the Paired group showed a $38.4 \%$ increase in responding for trial block 7 as compared with trial block 1. In contrast, leeches in the Backward Paired group showed a 39\% decrease in responding over their baseline levels. Thus, it appears that, as in Experiment 2, paired CS-US presentations result in an increase in the magnitude of the shortening reflex and that, at least under these conditions, leeches form associations when the CS and US are paired in a forward relationship but apparently do not form associations when the CS and US are paired in a backward relationship.

In addition to replicating the learning observed in Experiment 2 , the results of Experiment 3 provide a replication of the learning curve observed for leeches in the Paired group. That is, leeches in the Forward paired group of Experiment 3, just as leeches in the Paired group of Experiment 2, show a biphasic learning curve; responding remains stable across the first 5 blocks of training trials and then shows an increase in responding on blocks 6 and 7 .

\section{Experiment 4}

The goal of Experiment 4 was to replicate and extend our previous findings on the classical conditioning of the touch-elicited shortening reflex described in Experiments 2 and 3. Since our ultimate goal is to manipulate temporal parameters to study more complex aspects of associative learning as higher-order stimulus relations, including second-order conditioning, blocking, sensory preconditioning, and inhibitory learning, it is necessary to have precise control over the onset and offset of both the CS and the US. The manual application of the CS used in the previous 2 experiments does not allow for the exact control of either the CS presentation or the CS-US interval. For this reason, we have chosen to mimic the effect of the tactile CS by applying a 3 V $50 \mathrm{msec}$ pulse across the skin of the leech. Kristan et al. (1982) have used a similar procedure to study local bending. We use this procedure in this experiment to study classical conditioning of the touch-elicited shortening reflex.

In addition, we have used a random control group procedure (Rescorla, 1967) instead of the CS alone, US alone, and Unpaired groups we have employed previously. Rescorla (1967) suggested that only a random procedure that presents the CS and US to the animal with no predictive relationship between the 2 stimuli is optimal in assessing the effect of the predictive relation produced by the pairing operation. The regular presentations of the CS and/or US in the standard control groups provides the animal with the opportunity to learn about the stimuli and thcir relationship to each other and/or the training context. When stimuli occur in a random fashion this opportunity is not available.

\section{Materials and methods}

The procedures, animal selection, and apparatus were identical to those described in Experiment 3 except for the modifications described below. 


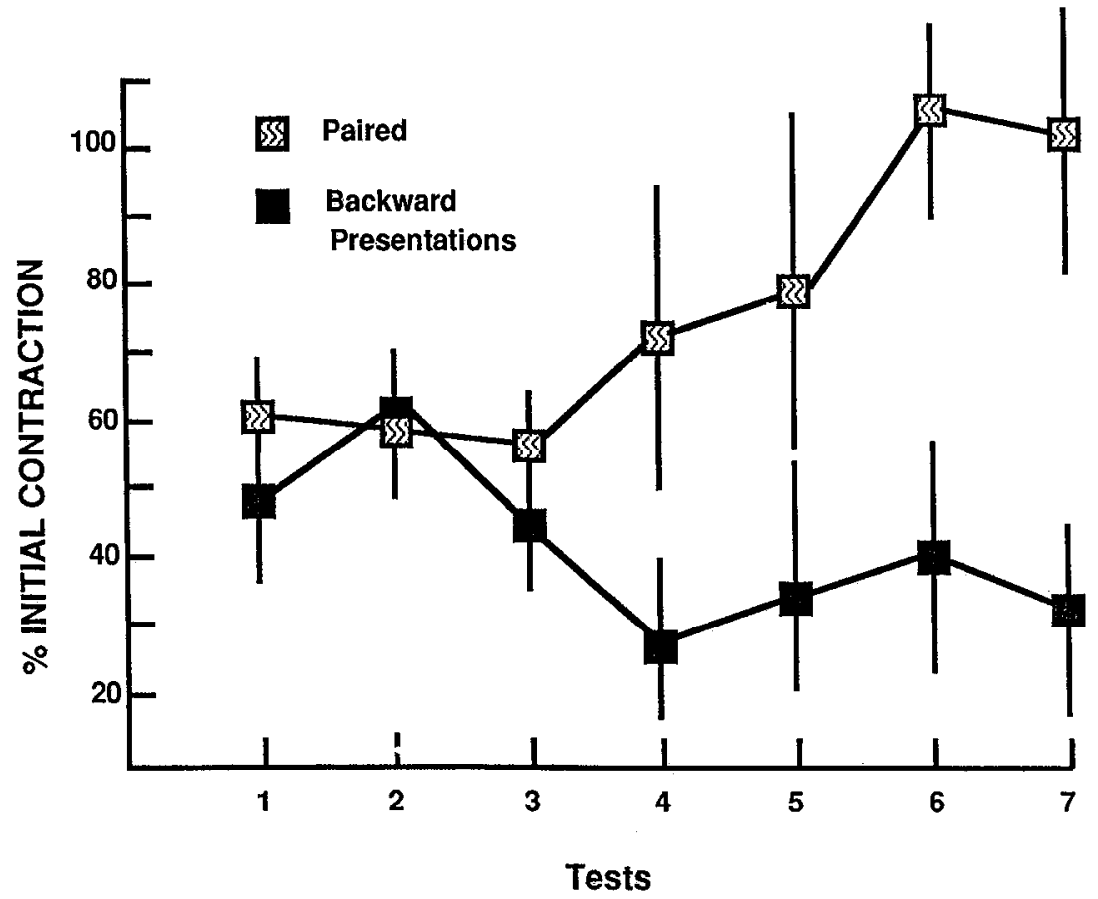

Figure 5. Mean percentage of initial contraction for animals that received forward paired presentations of the CS and US as compared with leeches that received backward paired presentations. Only the animals that received forward pairings showed an increase in responding to the touch stimulus.
Paired group $(P)$. Leeches in the paired group $(n=6)$ received 30 paired CS-US presentations, separated by a random ITI interval with a mean ITI of $2 \mathrm{~min}$.

Random group $(R)$. Leeches in the random group $(\mathrm{n}=6)$ received equal numbers of CS and US stimuli but in a predetermined random order. The ISI intervals were also arranged in a random order ranging from -400 msec CS-US presentation (backward relationship) to +4000 msec CS-US presentation in steps of approximately $500 \mathrm{msec}(-400$, $-100,+500$ then continuing at $500 \mathrm{msec}$ intervals).

The experiment was run in replications with 3 animals in each replication. Each animal was assigned randomly to either the Paired or the Random group by drawing straws. In alternative replications, 2 animals would be in the paired group and one in the random group and vice versa.

\section{Stimulus materials}

Two sets of bipolar electrodes, separated by approximately $7 \mathrm{~mm}$, spanned the body of the animal. The CS was delivered through the first set of electrodes and the US was delivered through the second set of electrodes via a Grass stimulator, model S88. The CS was a $3 \mathrm{~V}$ shock pulse, 50 msec in duration. The US was a $500 \mathrm{msec}$ train of $15 \mathrm{~V}$ pulses delivered at a rate of 10 pulses/sec. Leeches in the Paired group received 30 paired CS-US presentations with a delay ISI of approximately $400 \mathrm{msec}$. For leeches in the Random group, the ISIs ranged from 400 to 3000 msec, with the constraint that only one pairing (i.e., the CS and US occurring at the $400 \mathrm{msec}$ ISI) occur within each block of 5 trials. Thirty minutes prior to training, each animal was tested to determine its baseline responsiveness to the training stimulus, the stimulus was presented twice at a $15 \mathrm{~min}$ ISI. Following 30 training trials, the animals were tested again to assess the effect of the training.

\section{Dependent measure}

The leeches were scored as described in Experiment 2. In this experiment, though, the mean of the 2 prescores and the mean of the posttraining scores were used as the measure of the reflex responsiveness. Pre-post difference scores were calculated for leeches in each group.

\section{Statistical analysis}

Student's $t$ tests were used to evaluate the performance of the 2 groups. Between-group differences were assessed with independent $t$ tests, while within-group (pretraining vs posttraining performance) differences were assessed using paired $t$ tests.

\section{Results and discussion}

The mean percentage of initial contraction for the pre- and posttest trials for animals in each group is presented in Figure 6. As shown in Figure 6, leeches receiving paired CS-US presentations showed a significant increase in responding over their own bascline $[p(6)=3.56, p<0.05]$. In contrast, lecches in the Random control group showed a significant decrease in responding from their baseline $[p(6)=6.52, p<0.01]$. Leeches in the Random group showed a mean decrease of $68 \%$ in reflex amplitude compared with their baseline values, whereas leeches in the Paired group showed a $44 \%$ increase in responding over their baseline. A difference-scores analysis indicated that the difference between the 2 groups was significant, $t(10)=6.87, p$ $<0.001$. In addition, no differences were found in the pretest magnitude of the reflex between leeches in the 2 groups, $t(10)$ $=0.95, p>0.6$.

Thus, as in the previous 2 experiments, paired presentations of the CS and US result in an increased reflex amplitude compared with leeches in the control group.

\section{General Discussion}

The above series experiments demonstrate quite clearly that the leech is capable of associative classical conditioning. Following the pairing of a light tactile stimulus to the dorsal surface that results in local bending with a shock to the tail that reliably elicits stepping, leeches will learn to step to the touch. Moreover, in 3 experiments, the pairing of a light tactile stimulus to the head of the leech, which elicits the shortening reflex, with shock to the tail, which normally elicits a whole body contraction, resulted in an enhanced shortening response to the tactile stimulus. The learning is clearly pairing dependent since leeches that experienced the stimuli in unpaired, random, CS, or US alone configurations did not show an increase in either behavior. Moreover, as suggested by the extinction results of Experiment 3 , when the CS and US are no longer paired, a rapid decrease 


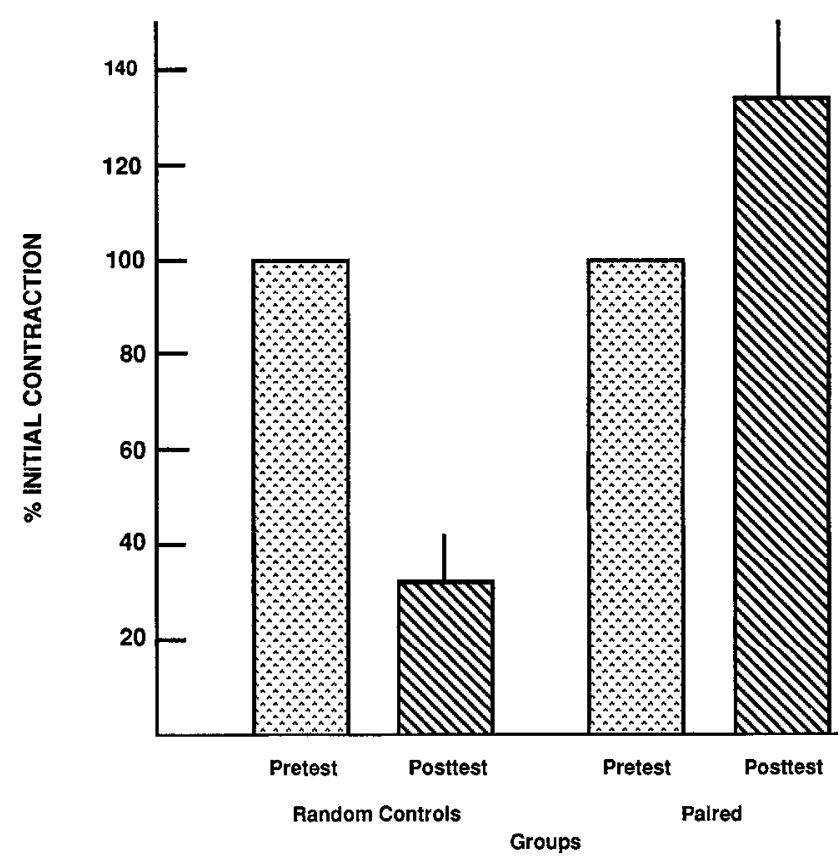

Figure 6. Pre- and posttraining scores for animals in the Paired and Random groups. The pretraining scores are normalized to $100 \%$ and the posttest scores are expressed as a percentage of initial contraction. Leeches in the Paired group showed a significant increase in responding, whereas leeches in the Random group showed a significant decrease in responding.

in conditioned responding is observed. We have no evidence for the rolc of contingency in this lcarning, but cxpcriments are in progress in the semi-intact preparation, and it appears that predictability is important for the formation of associations in the leech (Sahley, 1988). Moreover, predictability appears to be a fundamental characteristic of the associative process in the invertebrates Aplysia californica (Colwill, 1985; Hawkins et al., 1986), Hermissenda crassicornis (Farley, 1987a, b), and Limax maximus (Sahley et al., 1981b, 1984), as well as in vertebrates (Kamin, 1969; Rescorla, 1968, 1969).

The touch-elicited shortening reflex is also modified by habituation, dishabituation, and sensitization (Stoller and Sahley, 1985; Mazer et al., 1987). It has been suggested that these simpler forms of learning may be building blocks of the more complex associative learning (Humphrey, 1933; Hawkins and Kandel, 1984). Our data on classical conditioning of the shortening reflex is consistent with this hypothesis. That is, as seen in Figures 4 and 6, the learning curve of the paired animals shows 2 phases (slopes). The first phase from blocks 1-5 shows no significant increase or, more importantly, decrease in responding across these blocks of training. It is only late in training, blocks 6 and 7 , where a significant increase over baseline is observed. We suggest (but have not proved) that this indicates 2 phases for associative learning. The first phase appears as the prevention of habituation, perhaps in part via sensitization produced by the US, and a second phase in which the CR is facilitated ahove haseline. The notion of prevention of habituation is not new. Humphrey (1933) suggests that this may be involved in the associative process. Moreover, the diminished learning observed in animals experiencing CS preexposure points out the importance of the prevention of habituation to the subsequent formation of associations (Lubow and Moore, 1959; McDaniel and White, 1966; Lantz, 1973; Lubow, 1973).

It is clear from the classical conditioning experiments, as well as our sensitization experiments that the US has a sensitizing effect on the reflex (Boulis and Sahley, 1988). That is, leeches in the control groups, which experienced the US (US alone and Unpaired), showed a significantly slower rate of habituation compared with leeches in the CS-alone group. Leeches in the US-alone group do not show a significant decrement in responding until the fourth test, and leeches in the Unpaired group do not decrement significantly until the third test. Since the only difference between these 2 groups is the presence of repeated presentations of the CS in the Unpaired group, the faster habituation of leeches in this group is most probably due to habituation. In contrast, leeches in the CS-alone groups showed a dramatic decrement after only 2 blocks of training. Moreover, it is interesting to note that the US-alone group remains significantly more facilitated throughout training compared with leeches in the CS-alone and Unpaired groups. This suggests that sensitization produced by the US may be involved. However, we were surprised not to find a greater effect of sensitization, i.e., an enhancement of the reflex over baseline greater effect. In addition, we have some evidence that the behavioral facilitation resulting from a sensitizing or dishabituating stimulus is not immediately observed but rather takes several minutes to emerge (Boulis and Sahley, 1988). Thus, in the experimental design employed in the experiments reported in this paper we could be seeing the emergence of sensitization on an already somewhat habituated baseline. Further experiments are planned as direct tests of these hypotheses.

Our goal is to develop a semi-intact preparation that will show associative learning. It is critical to show causal events that result in the acquisition of learning or, in other words, the formation of an association, and it is important to monitor behavior throughout the experiment so that direct correlations between changes in neural events and behavior can be made. Moreover, it is essential to analyze the mechanisms underlying learning as it occurs in order to get at the associative mechanisms. Important cellular events may occur early in acquisition, and these would not be revealed in an end-point analysis. This is possible with the use of the semi-intact preparation we have developed. This type of preparation has been used to study habituation (Castellucci et al., 1970; Pinsker et al., 1970) and has also been used to study the neural correlates of associative learning in Limax maximus (Chang and Gelperin, 1980) and Aplysia californica (Lukowiak and Sahley, 1981; Lukowiak, 1986). Recent experiments in our lab indicate that these types of experiments may be feasible in the leech. Mazer et al. (1987) report that habituation, dishabituation, and sensitization in the semi-intact preparation are virtually identical to that observed in the intact animal. Moreover, pilot experiments indicate that classical conditioning of the shortening reflex appears to be a robust phenomenon in the semi-intact preparation (Sahley, 1988).

The experiments presented in the paper lay the foundation for further studies of associative learning in the leech, including higher-order phenomena such as blocking, second-order conditioning, and conditioned inhibition. Moreover, the study of learning in the leech provides an opportunity to determine the generalizability of the principles of learning to another phylum. Our goal is to use a combination of behavioral procedures that allow a clear definition of the variables that influence learning in the intact leech with an in vitro preparation that is amenable 
to behavioral and cellular analysis to determine the synaptic interactions and modifications important in the associative process.

\section{References}

Bagnoli, P., M. Brunelli, F. Magni, and M. Pellegrino (1975) The neuron of the fast conducting system in Hirudo medicinalis: Identification and synaptic connections with primary afferent neurons. Arch. Ital. Biol. 113: 21-43.

Baylor, D. A., and J. G. Nicholls (1969) Chemical and electrical synapses between cutaneous mechanoreceptors in the CNS of the leech. J. Physiol. 203: 591-609.

Belardetti, P., C. Biondi, L. Colombaioni, M. Brunelli, A. Trevisani, and C. Zavagno (1982) Role of serotonin and cyclic AMP on facilitation of the Fast Conducting System activity in the leech, Hirudo medicinalis. Brain Res. 246: 89-103.

Biondi, C., F. Berlladetti, M. Brunelli, A. Portolan, and A. Trevisani (1982) Increased synthesis of cyclic AMP and short-term plastic changes in the segmental ganglia of the leech, Hirudo medicinalis. Cell. Mol. Neurobiol. 2: 81-91.

Bitterman, M. E., R. Menzel, A. Fietz, and S. Schader (1983) Classical conditioning of proboscis-extension in honeybees. J. Comp. Physiol. Psychol. 97: 1007-1019.

Boulis, N., and C. L. Sahley (1988) A behavioral analysis of habituation and sensitization of shortening in the semi-intact leech. J. Neurosci. 8: 4621-4627.

Bruning, J. L., and B. L. Kintz (1968) Computational Handbook of Statistics., Scott, Foresman, Glenview, IL.

Byrne, J. H. (1987) Cellular analysis of associative learning. Physiol. Rev. 9: 435-487.

Carew, T. J., and C. L. Sahley (1986) Invertebrate learning and memory: From behavior to molecules. Annu. Rev. Neurosci. 9: 435-487.

Castellucci, V., H. Pinsker, I. Kupfermann, and E. R. Kandel (1970) Neuronal mechanisms of habituation and dishabituation of the gillwithdrawal reflex in Aplysia. Science 167: 1745-1748.

Chang, J. J., and A. Gelperin (1980) Rapid taste-aversion learning by an isolated molluscan central nervous system. Proc. Natl. Acad. Sci. USA 77: 6204-6206.

Colwill, R. M. (1985) Contex-US learning in Aplysia californica. Soc. Neurosci. Abstr. 11: 796.

Couvillon, P. A., and M. E. Bitterman (1980) Some phenomenon of associative learning in honeybees. J. Comp. Physiol. Psychol. 94: 878885 .

Couvillon, P. A., and M. E. Bitterman (1982) Compound conditioning in honeybees. J. Comp. Physiol. Psychol. 96: 192-199.

Crow, T. (1988) Cellular and molecular analysis of associative learning and memory in Hermissenda. Trends Neurosci. 11: 1988.

Debski, E. A., and W. O. Friesen (1985) Habituation of swimming activity in the medicinal leech. J. Exp. Biol. 116: 169-188.

DeRiemer, S. A., E. J. Elliott, E. R. Macagno, and K. J. Muller (1983) Morphological evidence that regenerating axons can fuse with severed axon segments. Brain Res. 272: 157-161.

Dickinson, M. H., and C. M. Lent (1984) Feeding behavior of the medicinal leech, Hirudo medicinalis. J. Comp. Physiol. A 154: 449455.

Dietzel, I. D., P. Drapeau, and J. G. Nicholls (1986) Voltage dependence of 5-hydroxytryptamine release at a synapse between identified leech neurones in culture. J. Physiol. 372: 191-205.

Dudai, Y. (1977) Properties of learning and memory in Drosophila melanogaster. J. Comp. Physiol. 114: 69-81.

Dudai, Y. (1985) Genes, enzymes and learning in Drosophila. Trends Neurosci. 8: 18-22.

Dudai, Y., Y.-N. Jan, D. Byers, W. G. Quinn, and S. Benzer (1976) Dunce, a mutant of Drosophila deficient in learning. Proc. Natl. Acad. Sci. USA 73: 1684-1688.

Erber, J. (1981) Neural correlates of learning in the honeybee. Trends Neurosci. 4: 270-273.

Erber, J. (1984) Response changes of single neurons during learning in the honeybee. In Primary Neural Substrates of Learning and Behavioral Change, D. L. Alkon and J. Farley, eds., pp. 275-285, Cambridge U. P., Cambridge, UK.

Erber, J., T. H. Masuhr, and R. Menzel (1980) Localization of shortterm memory in the brain of the bee, Apis mellifera. Physiol. Entomol. 5: 343-358.
Farley, J. (1987a) Contingency learning and causal detection in Hermissenda: I. Behavior. Behav. Neurosci. 101: 13-27.

Farley, J. (1987b) Contingency learning and causal detection in Hermissenda: II. Cellular mechanisms. Behav. Neurosci. 101:28-56.

Farley, J., and D. L. Alkon (1983) Changes in Hermissenda type B photoreceptors involving a voltage-dependent $\mathrm{Ca}^{++}$current during retention of associative learning. Soc. Neurosci. Abstr. 9: 167.

Farley, J., and D. L. Alkon (1985) Cellular mechanisms of learning, mcmory and information storage. Annu. Rev. Psychol. 36: 419-494.

French, K. A., and K. J. Muller (1986) Regeneration of a distinctive set of axosomatic contacts in the leech central nervous system. J. Neurosci. 6: 318-324.

Friesen, W. O. (1985) Neuronal control of leech swimming movements: Interactions between cell 60 and previously described oscillator neurons. J. Comp. Physiol. A 156: 231-242.

Friesen, W. O., and G. S. Stent (1978) Neural circuits for generating rhythmic movements. Annu. Rev. Biophys. Bioengin. 7: 37-61.

Friesen, W. O., G. S. Poon, and G. S. Stent (1978) Neuronal control of swimming in the medicinal leech. IV. Identification of a network of oscillatory interneurons. J. Exp. Biol. 75: 25-43.

Fuchs, P. A., J. G. Nicholls, and D. F. Ready (1981) Membrane properties and selective connexions of identified leech neurons in culturc. J. Physiol. 316: 203-223.

Fuchs, P. A., L. P. Henderson, and J. G. Nicholls (1982) Chemical transmission between individual Retzius and sensory neurones of the leech in culture. J. Physiol. 323: 195-210.

Galur, R., and S. H. Kindler (1966) Chemical specificity of the feeding response in Hirudo medicinalis. Comp. Biochem. Physiol. 17: 69-73.

Gormezano, I. (1972) Investigations of defense and reward conditioning in the rabbit. In Classical Conditioning, Vol. 2: Current Research and Theory, A. H. Black and W. F. Prokasy, eds., pp. 151181, Appleton, New York.

Hawkins, R. D., and E. R. Kandel (1984) Is there a cell-biological alphabet for simple forms of learning? Psychol. Rev. 91: 375-391.

Hawkins, R. D., T. J. Carew, and E. R. Kandel (1986) Effects of interstimulus interval and contingency on classical conditioning of the Aplysia siphon withdrawal reflex. J. Neurosci. 6: 1695-1701.

Henderson, T. B., and P. N. Strong (1972) Classical conditioning in the leech Macrobdella ditetra, as a function of CS and UCS intensity. Conditioned Reflex 7: 210-215.

Humphrey, G. (1933) The Nature of Learning in its Relation to the Living System, pp. 132-179, Harcourt, Brace, New York.

Jansen, J. K. S., and J. G. Nicholls (1972) Regeneration and changes in synaptic connections between individual nerve cells in the central nervous system of the leech. Proc. Natl. Acad. Sci. USA 69: 636639.

Kamin, L. J. (1969) Predictability, surprise, attention and conditioning. In Punishment and Aversive Behavior, R. Church and B. A. Campbell, eds., pp. 279-296, Appleton-Century-Crofts, New York.

Keppel, G. (1973) Design and Analysis: A Researcher's Handbook, pp. 283-344, 393-422, Prentice-Hall, Englewood Cliffs, N.J.

Kimble, G. A. (1961) Hilgard and Marquis' Conditioning and Learning, Appleton-Century-Crofts, New York.

Klein, M., J. Camardo, and E. R. Kandel (1982) Serotonin modulates a specific potassium current in the sensory neurons that show presynaptic facilitation in Aplysia. Proc. Natl. Acad. Sci. USA 79: 57135717.

Kramer, A. P. (1981) The nervous system of the glossiphonid leech Haementeria ghilianii. II. Synaptic pathways controlling body wall shortening. J. Comp. Physiol. 144: 449-457.

Kramer, A. P., and D. A. Weisblat (1985) Developmental neural kinship groups in the leech. J. Neurosci. 8: 388-407.

Kristan, W. B. (1982) Sensory and motor neurons responsible for the local bending response in leeches. J. Exp. Biol. 96: 161-181.

Kristan, W. B., Jr. (1983) The neurobiology of swimming in the leech. TINS 6: 84-88.

Kristan, W. B., Jr., and R. L. Calabrese (1976) Rhythmic swimming activity in neurons of the isolated nerve cord of the leech. J. Exp. Biol. 65: 329-354.

Kristan, W. B., S. McGirr, and S. Simpson (1982) Behavioral and mechanosensory neuron responses to skin stimulation in leeches. J. Exp. Biol. 94: 97-119.

Kristan, W. B., Jr., G. S. Stent, and C. A. Ort (1974) Neuronal control of swimming in the medicinal leech I. Dynamics of the swimming rhythm. J. Comp. Physiol. 94: 97-119. 
Lantz, A. (1973) Effect of number of trials, interstimulus interval, and dishabituation during CS habituation on subsequent conditioning in a CER paradigm. Anim. Learn. Behav. 4: 273-278.

Laverick, M. S. (1969) Mechanoreceptors, photoreceptors and rapid conduction pathways in the leech Hirudo medicinalis. J. Exp. Biol. 50: $129-140$.

Lockery, S. R., and W. Kristan (1985) Neural correlates of habituation and sensitization in the leech. Soc. Neurosci. Abstr. 11: 794.

Lockery, S. R., J. N. P. Rawlins, and J. A. Gray (1985) Habituation of the shortening reflex in the medicinal leech. Behav. Neurosci. 99: 333-341.

Loer, C. M., C. Schley, B. Zipser, and W. B. Kristan, Jr. (1986) Development of segmental differences in the pressure mechano-sensory neurons of the leech, Haementeria ghilanii. J. Comp. Neurol. 254: 403-409.

Lubow, R. E. (1973) Latent inhibition. Psychol. Bull. 79: 398-407.

Lubow, R. E., and A. U. Moore (1959) Latent inhibition: The effect of nonreinforced preexposure to the conditioned stimulus. J. Comp. Physiol. Psychol. 52: 415-419.

Lukowiak, K. (1986) In vitro classical conditioning of a gill withdrawal reflex in Aplysia: Neural correlates and possible neural mechanisms. J. Neurobiol, 17:83-101.

Lukowiak, K., and C. L. Sahley (1981) The in vitro classical conditioning of the gill withdrawal reflex of Aplysia californica. Science 212: 1516-1518

Macagno, E. R., K. J. Muller, and S. A. DeRiemer (1985) Regeneration of axons and synaptic connections by touch sensory neurons in the leech central nervous system. J. Neurosci. 5: 2510-2521.

Mackintosh, N. J. (1974) The Psychology of Animal Learning, Academic, New York.

Magni, F., and M. Pellegrino (1978a) Neural mechanisms underlying the segmental and generalized shortening reflexes in the leech. J. Comp. Physiol. 124: 339-351.

Magni, F., and M. Pellegrino (1978b) Patterns of activity and the effects of activation of the Fast Conducting System on the behavior of unrestrained leeches. J. Exp. Biol. 76: 123-135.

Mazer, J., N. Boulis, and C. L. Sahley (1987) Plasticity of the shortening reflex in the leech: Role of the FCS. Soc. Neurosci. Abstr. 13: 615.

McDaniel, J. W., and R. K. White (1966) A factorial study of the stimulus conditions of habituation. Perceptual Motor Skills 23: 259270.

Menzel, R. (1984) Short-term memory in bees. In Primary Neural Substrates of Learning and Behavioral Change, D. L. Alkon and J. Farley, eds., pp. 129-154, Cambridge U. P., Cambridge, UK.

Mpitsos, G. J., and K. Lukowiak (1985) Learning in gastropod molluscs. In The Mollusca, A. O. D. Willows, ed., pp. 95-267, Academic, New York.

Muller, K. J., and J. G. Nicholls (1973) Different properties of synapses between a single sensory neurone and two different motor cells in the leech C. N. S. J. Physiol. 238: 357-369.

Muller, K. J., and J. G. Nicholls (1981) Regeneration and plasticity. In Neurobiology of the Leech, K. J. Muller, J. G. Nicholls, and G. S. Stent, eds., pp. 197-226, Cold Spring Harbor Laboratory, Cold Spring Harbor, N.Y.

Muller, K. J., and S. A. Scott (1981) Transmission at a "direct" electrical connexion mediated by an interneuron in the leech. J. Physiol. 311: 565-583.

Nicholls, J. G., and B. A. Baylor (1968) Specific modalities and receptive fields of sensory neurons in the CNS of the leech. J. Neurophysiol. 31: 740-756.

Nicholls, J. G., and D. Purves (1970) Monosynaptic chemical and electrical connexions between sensory and motor cells in the nervous system of the leech. J. Physiol. 209: 647-667.

Nusbaum, M. P. (1986) Synaptic basis of swim initiation in the leech. III. Synaptic effects of serotonin-containing interneurones (cells 21 and 61) on swim CPG neurones (cells 18 and 208). J. Exp. Biol. 122 . 303-321.

Nusbaum, M. P., and W. B. Kristan (1986) Swim initiation in the leech by serotonin-containing interneurones, cells 21 and 61. J. Exp. Biol. 122: 277-302.

Ort, C. A., W. B. Kristan, Jr., and G. S. Stent (1974) Neuronal control of swimming in the medicinal leech. II. Identification and connections of motor neurons. J. Comp. Physiol. 94: 121-154.

Pinsker, H., L. Kupfermann, V. Castellucci, and E. R. Kandel (1970)
Habituation and dishabituation of the gill withdrawal reflex in Aplysia. Science 182: 1039-1042.

Quinn, W. G., P. P. Sziber, and R. Booker (1979) The Drosophila memory mutant amnesiac. Nature 277: 212-214.

Ready, D. F., and J. G. Nicholls (1979) Identified neurones isolated from leech CNS make selective connections in culture. Nature 281: 67-69.

Rescorla, R. A. (1967) Pavlovian conditioning and its proper control procedures. Psychol. Rev. 74: 71-80.

Rescorla, R. A. (1968) Probability of shock in the presence and absence of the CS in fear conditioning. J. Comp. Physiol. Psychol. 66: 1-5.

Rescorla, R. A. (1969) Conditioned inhibition of fear resulting in negative CS-US contingencies. J. Comp. Physiol. Psychol. 67: 504 509.

Sahley, C. L. (1984) Behavior theory and invertebrate learning. In Biology of Learning, P. Bateson and P. Marler, eds., Springer-Verlag, Berlin.

Sahley, C. L. (1988) Associative learning in the leech: Behavioral and cellular effects of predictability. Soc. Neurosci. Abstr. 14: 838 .

Sahley, C. L., and D. F. Ready (1985) Associative learning modifies two behaviors in the leech, Hirudo medicinalis. Soc. Neurosci. Abstr. 11: 367 .

Sahley, C. L., A. Gelperin, and J. W. Rudy (1981a) One trial learning modifies food odor preferences of a terrestrial mollusc. Proc. Natl. Acad. Sci. USA 78: 640-642.

Sahley, C. L., J. W. Rudy, and A. Gelperin (1981b) An analysis of associative learning in a terrestrial mollusc. I. Higher-order conditioning, blocking, and a transient US pre-exposure effect. J. Comp. Physiol. 144: 1-8.

Sahley, C. L., J. W. Rudy, and A. Gelperin (1984) Associative learning in a mollusc: A comparative analysis. In Primary Neural Substrates of Learning and Behavioral Change, D. L. Alkon and J. Farley, eds., pp. 243-258, Cambridge U. P., New York.

Scott, S. A., and K. J. Muller (1980) Synapse regeneration and signals for directed axonal growth in the C.N.S. of the leech. Dev. Biol. 80: 345-363.

Shankland, M., and D. A. Weisblat (1984) Stepwise commitment of blast cell fates during the positional specifications of the $O$ and $P$ cell lines in the leech. Dev. Biol. 106: 326-342.

Stent, G. S., and D. A. Weisblat (1985) Cell lineage in the development of invertebrate nervous systems. Annu. Rev. Neurosci. 8: 45-70.

Stern-Tomlinson, W., M. P. Nusbaum, L. E. Perez, and W. B. Kristan, Jr. (1986a) A quantitative study of the variants of crawling in the leech. Soc. Neurosci. Abstr. 11: 267.

Stern-Tomlinson, W., M. P. Nusbaum, L. E. Perez, and W. B. Kristan, Jr. (1986b) A kinemetic study of crawling behavior in the leech, Hirudo medicinalis. J. Comp. Physiol. A 158: 593-603.

Stewart, R. R., E. R. Macagno, and B. Zipser, (1985) The embryonic development of peripheral neurons in the body wall of the leech, Haemopis marmorata. Brain Res. 332: 150-157.

Stewart, R. R., D. Speigel, and E. R. Macagno (1986) Segmental differentiation in the leech nervous system: The genesis of cell number in the segmental ganglia. J. Comp. Neurol. 253: 253-259.

Stoller, D., and C. L. Sahley (1985) Habituation and sensitization of the shortening reflex in the leech, Hirudo medicinalis. Soc. Neurosci. Abstr. 11:367.

Stuart, A. E. (1970) Physiological and morphological properties of motorneurones in the central nervous system of the leech. J. Physiol. 209: 627-646.

Tempel, B. L., M. S. Livingstone, and W. G. Quinn (1984) Mutations in the dopadecarboxylase gene affect learning in Drosophila. Proc. Natl. Acad. Sci. USA 81: 3577-3581.

Tully, T. (1984) Drosophila learning: Behavior and biochemistry. Behav. Genet. 14: 527-557.

Tully, T. (1987) Drosophila learning and memory revisited. Trends Neurosci. 10: 330-335.

Weeks, J. C. (1982a) Segmental specialization of a leech swim-initiating interneuron, cell 205. J. Neurosci. 2: 972-985.

Weeks, J. C. (1982b) Synaptic basis of swim initiation in the leech. II. A pattern-generating neuron (cell 208) which mediates motor effects of swim-initiating neurons. J. Comp. Physiol. 148: 265-279.

Weeks, J. C., and W. B. Kristan, Jr. (1978) Initiation, maintenance and modulation of swimming in the medicinal leech by the activity of a single neurone. J. Exp. Biol. 77: 71-88. 